\title{
Measurement of the neutron electric form factor $\mathrm{G}_{\mathrm{E}}^{\mathrm{n}}$ in the reaction ${ }^{3} \overrightarrow{\mathbf{H e}}\left(\overrightarrow{\mathbf{e}}, \mathrm{e}^{\prime} \mathbf{n}\right)$
}

\author{
Björn Sören Schlimme* on behalf of the A1 collaboration \\ Johannes Gutenberg-Universität, Mainz \\ E-mail: schlimme@kph.uni-mainz.de
}

\begin{abstract}
The electric to magnetic form factor ratio of the neutron, $G_{\mathrm{E}}^{\mathrm{n}} / G_{\mathrm{M}}^{\mathrm{n}}$, has been determined from asymmetries measured in the reaction ${ }^{3} \overrightarrow{\mathrm{He}}\left(\overrightarrow{\mathrm{e}}, \mathrm{e}^{\prime} \mathrm{n}\right)$ in quasielastic kinematics at a momentum transfer of $Q^{2}=1.58 \mathrm{GeV}^{2} / c^{2}$. Since the magnetic form factor $G_{\mathrm{M}}^{\mathrm{n}}$ is well known, the electric form factor $G_{\mathrm{E}}^{\mathrm{n}}$ can be derived. The experiment was performed at the electron accelerator Mainz Microtron within the A1 spectrometer facility. Longitudinally polarized electrons were scattered on a high pressure polarized ${ }^{3} \mathrm{He}$ gas target. The scattered electrons were detected with a magnetic spectrometer, for detection of the recoiling neutrons a dedicated neutron detector was used.
\end{abstract}

Sixth International Conference on Quarks and Nuclear Physics

April 16-20, 2012

Ecole Polytechnique, Palaiseau, Paris

\footnotetext{
* Speaker.
} 


\section{Electromagnetic nucleon form factors}

The electromagnetic structure of the nucleon can be studied in elastic electron scattering. To lowest order, the electromagnetic interaction between the electron and the nucleon can be described by the exchange of a single virtual photon with invariant mass squared $q^{2}=\omega^{2}-\vec{q}^{2} \equiv-Q^{2}$, with the three-momentum transfer $\vec{q}$ and the energy transfer $\omega$. Two form factors (FF) can be introduced to parametrize the scattering cross section. In a particular parametrization, the electric and magnetic Sachs FF $G_{\mathrm{E}}\left(Q^{2}\right)$ and $G_{\mathrm{M}}\left(Q^{2}\right)$ contain information on the charge and magnetization densities, respectively (see for instance [1]). Precise measurements over a large $Q^{2}$ range can be used to test nonperturbative QCD and to constrain phenomenological models of the nucleon structure.

\section{Measurement technique}

The experimental determination of the neutron FF is hindered compared to the determination of the proton FF due to the absence of a free neutron target. To investigate the neutron FF, scattering experiments on light nuclei like deuteron and ${ }^{3} \mathrm{He}$ can be exploited. Certainly the use of these targets introduces several additional challenges. The presence of the protons contaminates the interesting observables. Also nuclear binding effects have to be considered.

Actually the magnetic FF of the neutron, $G_{\mathrm{M}}^{\mathrm{n}}$, has been determined with a precision in the order of a few percent up to moderate high $Q^{2}$ values (see [2] and references there). On the other hand, the precision one can achieve for $G_{\mathrm{E}}^{\mathrm{n}}$, the neutron electric $\mathrm{FF}$, in unpolarized quasielastic scattering is extremely limited: Since $G_{\mathrm{E}}^{\mathrm{n}}$ is much smaller than $G_{\mathrm{M}}^{\mathrm{n}}$ the unpolarized cross section is strongly dominated by the contribution of the magnetic FF in that case. Alternatively one can use techniques involving polarization degrees of freedom. Observables in these measurements can be particularly sensitive to the FF ratio $G_{\mathrm{E}}^{\mathrm{n}} / G_{\mathrm{M}}^{\mathrm{n}}$. Since $G_{\mathrm{M}}^{\mathrm{n}}$ is well known, one can obtain $G_{\mathrm{E}}^{\mathrm{n}}$.

The experiment discussed here is based on the analysis of cross section asymmetries of the fundamental process $\vec{n}\left(\vec{e}, e^{\prime} n\right)$ where longitudinally polarized electrons scatter on polarized neutrons. Again, since there is no free neutron target, a polarized ${ }^{3} \mathrm{He}$ target was used instead. Due to its special spin structure, polarized ${ }^{3} \mathrm{He}$ can serve as an effective polarized neutron target [3]. The mean polarization of the neutrons and the protons relative to the polarization of ${ }^{3} \mathrm{He}$ is $0.86 \pm 0.02$ and $-0.028 \pm 0.004$, respectively [4]. For a separation between contributions from quasielastic scattering on the neutron and on the proton, the scattered electrons were detected in coincidence with the recoil nucleons. By means of veto layers, the neutron detector had the capability to distinguish between neutrons and protons.

Comparing count rates for different helicities of the incoming electron, one can observe the beam helicity asymmetry $A=\frac{N^{+}-N^{-}}{N^{+}+N^{-}}$with the luminosity corrected count rates $N^{+}$(electron spin orientated parallel to the direction of the electron beam) and $N^{-}$(antiparallel). For a free neutron at rest the asymmetry is given in the one photon exchange approximation through [5]

$$
A=\frac{a G_{\mathrm{E}}^{\mathrm{n}} G_{\mathrm{M}}^{\mathrm{n}} \sin \left(\theta^{*}\right) \cos \left(\phi^{*}\right)+b G_{\mathrm{M}}^{\mathrm{n} 2} \cos \left(\theta^{*}\right)}{c G_{\mathrm{E}}^{\mathrm{n}}+d G_{\mathrm{M}}^{\mathrm{n}} 2} \cdot P_{\mathrm{e}} P_{\mathrm{n}} .
$$

The kinematic factors $a, b, c$ and $d$ are of similar magnitude for the kinematics of this experiment, $P_{\mathrm{e}}$ and $P_{\mathrm{n}}$ are the electron and the neutron polarizations, respectively. The angles $\theta^{*}$ and $\phi^{*}$ specify the neutron polarization vector, see fig. 1 . 

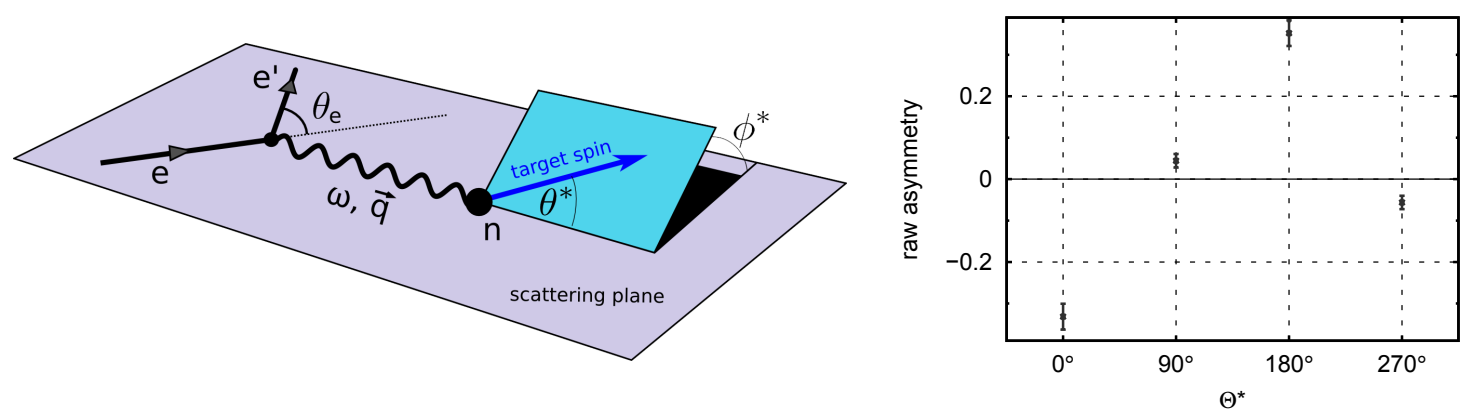

Figure 1: Left: Definition of the polarization orientation angles $\theta^{*}$ and $\phi^{*}$ relative to the momentum transfer $\vec{q}$ and the scattering plane. Right: Raw experimental asymmetries of events from the reaction ${ }^{3} \mathrm{He}\left(\overrightarrow{\mathrm{e}}, \mathrm{e}^{\prime} \mathrm{n}\right)$ for different target polarization orientations (with $\phi^{*}=0$ ). The asymmetries for $\theta^{*}=90^{\circ}$ and $270^{\circ}$ are sensitive to $\frac{G_{\mathrm{E}}^{\mathrm{n}}}{G_{\mathrm{M}}^{\mathrm{n}}}$, the asymmetries for $\theta^{*}=0^{\circ}$ and $180^{\circ}$ have been measured for a reduction of systematics.

As is evident from (2.1) $A$ is sensitive to $\frac{G_{\mathrm{E}}^{\mathrm{n}}}{G_{\mathrm{M}}^{\mathrm{n}}}$ for a target polarization orientated in the scattering plane and perpendicular to the momentum transfer (for instance $\theta^{*}=90^{\circ}$ and $\phi^{*}=0^{\circ}$, labeled as $A_{\perp}$ ). A supplemental measurement of the asymmetry $A_{\|}$for a target polarization aligned with the momentum transfer $\left(\theta^{*}=0^{\circ}\right)$ allows for the calculation of the asymmetry ratio

$$
\frac{A_{\perp}}{A_{\|}} \propto \frac{a}{b} \cdot \frac{\left[P_{\mathrm{e}} P_{\mathrm{n}}\right]_{\perp}}{\left[P_{\mathrm{e}} P_{\mathrm{n}}\right]_{\|}} \cdot \frac{G_{\mathrm{E}}^{\mathrm{n}}}{G_{\mathrm{M}}^{\mathrm{n}}} .
$$

In this case, only relative polarizations have to be determined and several dilution factors, which effect the single asymmetries, tend to cancel in the ratio. For reasons of redundance, asymmetries were also measured for $\left(\theta^{*}=180^{\circ}, \phi^{*}=0^{\circ}\right)$ and $\left(\theta^{*}=270^{\circ}, \phi^{*}=0^{\circ}\right)$.

\section{Experimental setup}

The experiment was carried out at the electron accelerator facility MAMI [6, 7]. MAMI provided $1.508 \mathrm{GeV}$ longitudinally polarized electrons with a beam current around $10 \mu \mathrm{A}$. The beam polarization was measured twice a day using a møller polarimeter. Only minor fluctuations were found around a mean polarization of $76.3 \%$.

The experiment was set up in the spectrometer hall of the A1 collaboration [8]. The scattered electrons were detected under a central scattering angle of $78.6^{\circ}$ with Spektrometer A, a high resolution magnetic spectrometer. Its detector system comprises of vertical drift chambers, scintillator paddles and a Čerenkov threshold detector for the distinction between electrons and pions.

For the detection of the recoiling nucleons a plastic scintillator array was used. It consisted of six layers with five bars. Each bar $\left(500 \times 100 \times 100 \mathrm{~mm}^{3}\right)$ was equipped with two photomultiplier tubes. Two $10 \mathrm{~mm}$ thin veto layers were installed additionally in front of the detector for charged particle rejection. The neutron detector was heavily shielded except for the entrance window pointing to the target. The latter was only shielded with $1 \mathrm{~cm}$ lead to keep the proton to neutron conversion probability small for protons coming from the target.

As target a highly polarized 5 bar ${ }^{3} \mathrm{He}$ gas target was used [9]. The target cells were put in a magnetically shielded target box which provided a magnetic guiding field. By altering the direction 
of this field, the direction of the target polarization could be freely oriented. The relaxation time was about 30 to 40 hours under beam conditions. The target cell was changed twice a day resulting in a mean target polarization of $55.6 \%$.

\section{Analysis}

The raw data of the individual detectors are considered for reconstruction of kinematic quantities of the detected particles. Electron identification is mainly accomplished through the evaluation of the Čerenkov detector signal. A coincidence time resolution of $2.1 \mathrm{~ns}$ (FWHM) between the electron and the neutron arm is achieved, the fraction of accidental coincidences is estimated to be $0.7 \%$.

For a suppression of inelastic events kinematical cuts are applied. Veto counters of the neutron detector are used for a rejection of charged particles. The number of protons which are misidentified as neutrons is estimated by the analysis of data taken on a hydrogen target. The relative fraction is found to be $(12.8 \pm 1.7) \%$. Since the polarization of the protons relative to the target polarization is comparatively small, the main effect of the proton background is a dilution of the single asymmetries in the order of $10 \%$. The asymmetry ratio, which is used to extract $\frac{G_{\mathrm{E}}^{\mathrm{n}}}{G_{\mathrm{M}}^{\mathrm{n}}}$, is effected much less. Using events identified as quasielastic scattering on the proton, the asymmetries of the proton background can be assessed. The correction for the proton contribution is estimated to be $(1.6 \pm 1.2) \%$.

Once the data selection has been performed, the analysis is based on the study of the measured asymmetries of the selected ${ }^{3} \mathrm{He}\left(\overrightarrow{\mathrm{e}}, \mathrm{e}^{\prime} \mathrm{n}\right)$ events. The raw experimental asymmetries are shown in fig. 1 for the four target spin orientations. For a good handle on variations of kinematics, present beam and target polarizations, which effect the asymmetry (2.1), an event-by-event analysis based on a maximum likelihood fit is performed. The influence of effects like detector resolutions and energy loss of the particles is investigated using a Monte Carlo simulation. Different contributions to the systematic uncertainty of $\frac{G_{\mathrm{E}}^{\mathrm{n}}}{G_{\mathrm{M}}^{\mathrm{n}}}$ add up to $6.4 \%$ relative, while the statistical error is $23 \%$. Corrections due to the effects of final state interactions are not included yet, they will be estimated by using the general eikonal approximation [10]. They are expected to be in the order of only a few percent. For a determination of $G_{\mathrm{E}}^{\mathrm{n}}, G_{\mathrm{M}}^{\mathrm{n}}$ data from [2] are used. The result of the analysis is shown in fig. 2.

\section{Summary and Outlook}

A measurement of the neutron electric form factor has been performed at the Mainz Microtron in 2008. The reaction ${ }^{3} \overrightarrow{\mathrm{He}}\left(\overrightarrow{\mathrm{e}}, \mathrm{e}^{\prime} \mathrm{n}\right)$ has been studied. Beam helicity asymmetries for a target polarization aligned perpendicular to the momentum transfer are sensitive to the form factor ratio $\frac{G_{\mathrm{E}}^{\mathrm{n}}}{G_{\mathrm{M}}^{\mathrm{n}}}$. Supplemental asymmetries for parallel orientation are used for a reduction of systematics. Using existing data for $G_{\mathrm{M}}^{\mathrm{n}}, G_{\mathrm{E}}^{\mathrm{n}}$ is determined. The analysis is almost finished, but nuclear corrections still have to be applied.

Currently a new highly segmented, high performance neutron detector is being developed within the A1 collaboration. This detector will be used in a systematic neutron form factor measurement campaign in the $Q^{2}$ range from 0.2 to $1.5(\mathrm{GeV} / c)^{2}$ [26]. 


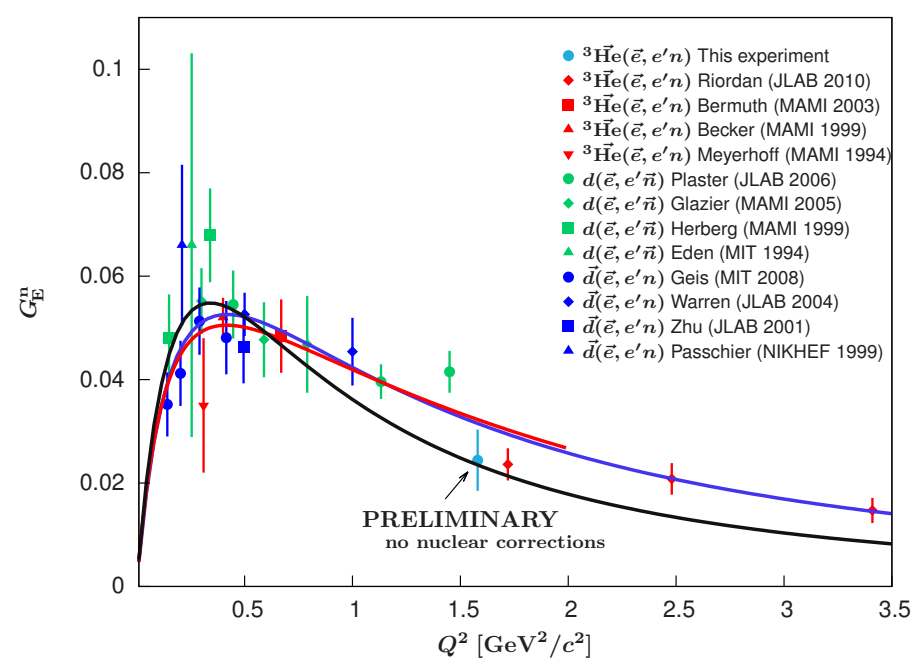

Figure 2: (color online). The preliminary result for $G_{\mathrm{E}}^{\mathrm{n}}$ of this experiment (light blue) compared to data from other double polarization experiments [11-22]. Red data points are from experiments using the reaction ${ }^{3} \mathrm{He}\left(\overrightarrow{\mathrm{e}}, \mathrm{e}^{\prime} \mathrm{n}\right)$. The experiments belonging to the blue data points made use of a polarized deuteron target. The results shown as green data points were obtained by measuring the neutron recoil polarization instead of having a polarized target. The error bars shown are statistical and systematic uncertainties added in quadrature. Shown is also the result of a two-dipole fit [23] (blue line), the result of a dispersion analysis [24] (red line) and the so-called Galster parametrization [25] (black line).

\section{Acknowledgments}

We gratefully acknowledge the essential support from the technical staff at the Mainz Microtron and thank the accelerator group for the excellent beam quality. Work supported by the "Deutsche Forschungsgemeinschaft (DFG)" with a Collaborative Research Centre (SFB 443).

\section{References}

[1] C.F. Perdrisat, V. Punjabi and M. Vanderhaeghen, Nucleon electromagnetic form factors, Prog. Part. Nucl. Phys. 59 (2007) 694.

[2] J. Lachniet et al., Precise Measurement of the Neutron Magnetic Form Factor $G_{M}^{n}$ in the Few-GeV Region, Phys. Rev. Lett. 102 (2009) 192001.

[3] B. Blankleider and R.M. Woloshyn, Quasi-elastic scattering of polarized electrons on polarized ${ }^{3} H e$, Phys. Rev. C 29 (1984) 538.

[4] S. Scopetta, Neutron single spin asymmetries from semi-inclusive deep inelastic scattering off transversely polarized ${ }^{3}$ He, Phys. Rev. D 75 (2007) 054005.

[5] T.W. Donelly and A.S. Raskin, Considerations of Polarization in Inclusive Electron Scattering from Nuclei, Ann. Phys. 169 (1986) 247.

[6] H. Herminghaus et al., The Design of a Cascaded 800-MeV Normal Conducting CW Racetrack Microtron, Nucl. Instrum. Methods 138 (1976) 1.

[7] K.H. Kaiser et al., The 1.5-GeV harmonic double-sided microtron at Mainz University, Nucl. Instrum. Methods A 593 (2008) 159. 
[8] K.I. Blomqvist et al., The three-spectrometer facility at the Mainz microtron MAMI, Nucl. Instrum. Methods A 403 (1998) 263.

[9] J. Krimmer et al., A highly polarized He-3 target for the electron beam at MAMI, Nucl. Instrum. Methods A 611 (2009) 18.

[10] M. Sargsian, Selected Topics in High Energy Semi-Exclusive Electro-Nuclear Reactions, Int. J. Mod. Phys. E 10 (2001) 405.

[11] S. Riordan et al., Measurements of the Electric Form Factor of the Neutron up to $Q^{2}=3.4 \mathrm{GeV}^{2}$ Using the Reaction ${ }^{3} \overrightarrow{\mathrm{He}}\left(\vec{e}, e^{\prime} n\right) p p$, Phys. Rev. Lett. 105 (2010) 262302.

[12] J. Bermuth et al., The neutron charge form factor and target analyzing powers from ${ }^{3} \overrightarrow{\mathrm{He}}\left(\overrightarrow{\mathrm{e}}, \mathrm{e}^{\prime} \mathrm{n}\right)$ scattering, Phys. Lett. B 564 (2003) 199.

[13] J. Becker et al., Determination of the neutron electric form factor from the reaction ${ }^{3} \mathrm{He}\left(e, e^{\prime} n\right)$ at medium momentum transfer, Eur. Phys. J. A 6 (1999) 329.

[14] M. Meyerhoff et al., First measurement of the electric formfactor of the neutron in the exclusive quasielastic scattering of polarized electrons from polarized ${ }^{3}$ He, Phys. Lett. B 327 (1994) 201.

[15] B. Plaster et al., Measurements of the neutron electric to magnetic form factor ratio $G_{E n} / G_{M n}$ via the ${ }^{2} \mathrm{H}\left(\vec{e}, e^{\prime} \vec{n}\right){ }^{1} \mathrm{H}$ reaction to $Q^{2}=1.45(\mathrm{GeV} / \mathrm{c})^{2}$, Phys. Rev. C 73 (2006) 025205.

[16] D.I. Glazier et al., Measurement of the electric form factor of the neutron at $Q^{2}=0.3-0.8(\mathrm{GeV} / \mathrm{c})^{2}$, Eur. Phys. J. A 24 (2005) 101.

[17] C. Herberg et al., Determination of the neutron electric form factor in the $D\left(e, e^{\prime} n\right) p$ reaction and the influence of nuclear binding, Eur. Phys. J. A 5 (1999) 131.

[18] T. Eden et al., Electric form factor of the neutron from the ${ }^{2} H\left(\vec{e}, e^{\prime} \vec{n}\right)^{1} H$ reaction at $Q^{2}=0.255(\mathrm{GeV} / \mathrm{c})^{2}$, Phys. Rev. C 50 (1994) R1749.

[19] E. Geis et al., Charge Form Factor of the Neutron at Low Momentum Transfer from the ${ }^{2} \overrightarrow{\mathrm{H}}\left(\vec{e}, e^{\prime} n\right)^{1} \mathrm{H}$ Reaction, Phys. Rev. Lett. 101 (2008) 042501.

[20] G. Warren et al., Measurement of the Electric Form Factor of the Neutron at $Q^{2}=0.5$ and 1.0 $G e V^{2} / c^{2}$, Phys. Rev. Lett. 92 (2004) 042301.

[21] H. Zhu et al., Measurement of the Electric Form Factor of the Neutron through $\vec{d}\left(\vec{e}, e^{\prime} n\right) p$ at $Q^{2}=0.5(\mathrm{GeV} / \mathrm{c})^{2}$, Phys. Rev. Lett. 87 (2001) 081801.

[22] I. Passchier et al., Charge Form Factor of the Neutron from the Reaction ${ }^{2} \overrightarrow{\mathrm{H}}\left(\vec{e}, e^{\prime} n\right) p$, Phys. Rev. Lett. 82 (1999) 4988.

[23] T.R. Gentile and C.B. Crawford, Neutron charge radius and the neutron electric form factor, Phys. Rev. C 83 (2011) 055203.

[24] M.A. Belushkin, H.W. Hammer and U.G. Meissner, Dispersion analysis of the nucleon form factors including meson continua, Phys. Rev. C 75 (2007) 035202.

[25] S. Galster et al., Elastic electron-deuteron scattering and the electric neutron form factor at four-momentum transfers $5 \mathrm{fm}^{-2}<q^{2}<14 \mathrm{fm}^{-2}$, Nucl. Phys. B 32 (1971) 221.

[26] A. Denig, M. Vanderhaeghen et al., The Low-Energy Frontier of the Standard Model, Proposal for the establishment and funding of Collaborative Research Centre 1044 (2011). 